\title{
The population of hot subdwarf stars studied with Gaia
}

\section{The catalog of known hot subdwarf stars ${ }^{\star}$}

\author{
S. Geier ${ }^{1,2,3}$, R. H. Østensen ${ }^{4}$, P. Nemeth ${ }^{3}$, N. P. Gentile Fusillo², B. T. Gänsicke ${ }^{2}$, J. H. Telting ${ }^{5}$, \\ E. M. Green ${ }^{6}$, and J. Schaffenroth ${ }^{3}$
}

\author{
${ }^{1}$ Institute for Astronomy and Astrophysics, Kepler Center for Astro and Particle Physics, Eberhard Karls University, Sand 1, \\ 72076 Tübingen, Germany \\ e-mail: geier@astro.uni-tuebingen.de \\ 2 Department of Physics, University of Warwick, Coventry CV4 7AL, UK \\ 3 Dr. Karl Remeis-Observatory \& ECAP, Astronomical Institute, Friedrich-Alexander University Erlangen-Nuremberg, \\ Sternwartstr. 7, 96049 Bamberg, Germany \\ 4 Department of Physics, Astronomy, and Materials Science, Missouri State University, Springfield, MO 65804, USA \\ 5 Nordic Optical Telescope, Rambla José Ana Fernández Pérez 7, 38711 Brena Baja, Spain \\ ${ }^{6}$ Steward Observatory, University of Arizona, 933 North Cherry Avenue, Tucson, AZ 85721, USA
}

Received 25 November 2016 / Accepted 9 December 2016

\begin{abstract}
In preparation for the upcoming all-sky data releases of the Gaia mission we compiled a catalog of known hot subdwarf stars and candidates drawn from the literature and yet unpublished databases. The catalog contains 5613 unique sources and provides multiband photometry from the ultraviolet to the far infrared, ground based proper motions, classifications based on spectroscopy and colors, published atmospheric parameters, radial velocities and light curve variability information. Using several different techniques we removed outliers and misclassified objects. By matching this catalog with astrometric and photometric data from the Gaia mission, we will develop selection criteria to construct a homogeneous, magnitude-limited all-sky catalog of hot subdwarf stars based on Gaia data.
\end{abstract}

Key words. subdwarfs - stars: horizontal-branch

\section{Introduction}

Hot subdwarf stars ( $\mathrm{sdO} / \mathrm{Bs})$ have spectra similar to main sequence $\mathrm{O} / \mathrm{B}$ stars, but are subluminous and more compact. The formation and evolution of those objects is still unclear. In the Hertzsprung-Russell diagram those stars are located at the blueward extension of the horizontal branch (HB), the so called extreme or extended horizontal branch (EHB, Heber et al. 1986) and are therefore considered to be core helium-burning stars.

To end up on the EHB, stars have to lose almost their entire hydrogen envelopes in the red-giant phase most likely via binary mass transfer. Hot subdwarfs turned out to be important objects to study close binary interactions and their companions can be planets, brown dwarfs, all kinds of main sequence stars, white dwarfs, and maybe even neutron stars or black holes. Hot subdwarf binaries with massive white dwarf companions are candidates for the progenitors of type Ia supernovae. They are possibly ejected by such supernovae as hypervelocity stars (see Geier 2015). Hot subdwarfs dominate old stellar populations in blue and ultraviolet bands. Their atmospheres are peculiar and can be used to study diffusion processes, such as gravitational settling or radiative levitation. Furthermore, several types of pulsating sdO/Bs have been found and turn out to be well suited for asteroseismic analyses. For a comprehensive review of the state-of-the-art hot subdwarf research see Heber (2016).

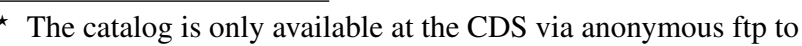
cdsarc.u-strasbg.fr (130.79.128.5) or via http://cdsarc.u-strasbg.fr/viz-bin/qcat?]/A+A/600/A50
}

$\mathrm{SdO} / \mathrm{B}$ stars were initially found in surveys looking for faint blue stars at high Galactic latitudes (Humason \& Zwicky 1947). The first larger-area surveys for such objects were the Tonantzintla survey (TON, Iriarte \& Chavira 1957; Chavira 1958, 1959), the Palomar Haro Luyten survey (PHL, Haro \& Luyten 1962), and the Palomar-Green (PG) survey (Green et al. 1986). The Kitt Peak-Downes (KPD) survey covered a substantial area in the Galactic plane for the first time (Downes 1986). Collecting these early discoveries Kilkenny et al. (1988) published the first catalog of spectroscopically identified hot subdwarf stars. This catalog contained photometry, spectral types and some atmospheric parameters for the $1225 \mathrm{sdO} / \mathrm{Bs}$ known at that time.

Many more hot subdwarfs have been detected subsequently in the Hamburg Quasar Survey (HS, Hagen et al. 1995), the Hamburg ESO survey (HE, Wisotzki et al. 1996), the EdinburghCape Survey (EC, Stobie et al. 1997) and the Byurakan surveys (FBS, SBS, Mickaelian et al. 2007, 2008). Østensen (2006) did an extensive literature search and created the hot subdwarf database with state-of-art interface linking essentially all the information available in the archives for more than 2300 stars. This database is still widely used in the field.

However, since 2006 the number of known hot subdwarfs again increased by a factor of more than two. The Sloan Digital Sky Survey (SDSS) provided spectra of almost $2000 \mathrm{sdO} / \mathrm{Bs}$ (Geier et al. 2015b; Kepler et al. 2015, 2016), reaching down to much fainter magnitudes than previous surveys. On the bright 
end of the magnitude distribution, new samples of hot subdwarfs have been selected from the EC survey and the GALEX all-sky survey photometry in the UV (e.g. Vennes et al. 2011). Furthermore, new large-area photometric and astrometric surveys have been and are currently conducted in multiple bands from the UV to the far infrared. Given this wealth of new high quality data, we consider it timely to compile a new catalog of hot subdwarf stars.

This catalog will be used as input and a calibration dataset to select a magnitude-limited, homogeneous, all-sky catalog of hot subdwarf stars using astrometry and photometry from the Gaia mission, which will allow us to study the properties of the hot subdwarf population with unprecedented accuracy.

\section{Constructing the catalog}

\subsection{Input data}

The basic data source for the catalog was the sample of hot subdwarfs classified as sdO/B from the database of Østensen (2006), which we consider fairly complete up to the date of publication. We added the subdwarf candidates from the FBS survey (Mickaelian et al. 2008), the sample of hot subdwarfs identified in the course of the Kepler mission (Østensen et al. 2010b), the large sample of sdO/Bs spectroscopically identified from the SDSS DR7 during the Massive Unseen Companions to Hot Faint Underluminous Stars from SDSS project (MUCHFUSS, Geier et al. 2015b), a yet unpublished sample of spectroscopically classified sdO/Bs selected from SDSS DR8-10, and the most recently published sdO/B candidates from SDSS DR12 (Kepler et al. 2016). We also included the candidate sample from SDSS DR10 classified as narrow-line hydrogen stars (NLHS) by Gentile Fusillo et al. (2015). The recently published large sample of $\mathrm{sdO} / \mathrm{Bs}$ from the complete EC survey was included as well and is important because of its location in the otherwise under-represented Southern hemisphere (Stobie et al. 1997; O'Donoghue et al. 2013; Kilkenny et al. 2015, 2016).

An important part of our catalog consists of a sample of several hundred yet unpublished sdO/Bs selected from GALEX, GSC, and 2MASS photometry by R. H. Østensen and E. M. Green, which have been classified based on follow-up spectroscopy taken with the INT/IDS, NOT/ALFOSC, WHT/ISIS, CAHA/TWIN, ESO-NTT/EFOSC2, and 4m-KPNO/RC spectrographs.

In addition, we added the sample of $\mathrm{sdO} / \mathrm{Bs}$ selected from the Guide Star and the Galaxy Evolution Explorer (GALEX) catalogs by Vennes et al. (2011), the samples of Oreiro et al. (2011) and Perez-Fernandez et al. (2016) selected using Virtual observatory tools and multiband photometry, and the first sample of sdO/Bs discovered by the Large Sky Area Multi-Object Fibre Spectroscopic Telescope (LAMOST) survey (Luo et al. 2016). Those samples are considered the most important ones to date and should cover more than $90 \%$ of the currently known sdO/Bs in the field. The growing number of hot subdwarf stars found in globular clusters has not been included in this release of the catalog.

\subsection{Multi-band photometry}

Using TOPCAT's (Taylor 2005) internal cross-match with a radius of 10 arcsec, we identified several hundred duplications and constructed a catalog of unique sources. To obtain homogeneous multi-band photometry, we cross-matched those objects again using a radius of 10 arcsec with well calibrated, large-area survey catalogs. Near-UV (NUV) and far-UV (FUV) photometry were

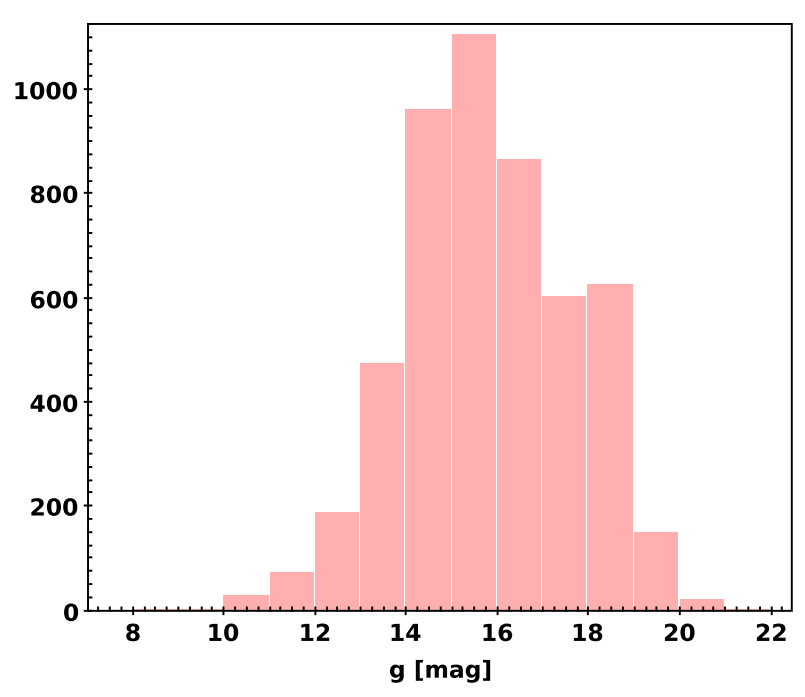

Fig. 1. Magnitude distribution of a representative catalog subset in the $g$-band. Photometry has been taken from SDSS if available, or otherwise from APASS, which also covers the brighter objects.

taken from the GALEX DR5 All-sky Imaging Survey (AIS, Bianchi et al. 2011). To account for the known systematic shifts of the GALEX magnitudes of bright targets we applied the corrections suggested by Camarota \& Holberg (2014).

Optical photometry was obtained from the Guide Star catalog (GSC 2.3.2, Lasker et al. 2008) in the $R_{\mathrm{F}} B_{\mathrm{J}} V I_{\mathrm{N}} B$-bands, the AAVSO Photometric All Sky Survey (APASS DR9, Henden et al. 2016) in the VBgri-bands, the SDSS DR12 (Alam et al. 2015) in the ugriz-bands, the VST-ATLAS (DR2, Shanks et al. 2015) and the Kilo-Degree (KiDS DR2, de Jong et al. 2015) ESO public surveys in the ugriz-bands. For the optical magnitude distribution of the catalog see Fig. 1.

Near Infrared photometry was obtained from the 2MASS All-Sky Catalog of Point Sources (Skrutskie et al. 2006) in the $J H K$-bands, the UKIRT Infrared Deep Sky Survey (UKIDSS Large Area Survey DR9, Lawrence et al. 2007) in the $Y J H K$ bands, the VISTA Hemisphere (VHS DR2, McMahon et al. in prep.), and the VISTA Kilo-degree Infrared Galaxy (VIKING DR4, Edge et al. 2013) ESO public surveys in the $Z Y J H K_{\mathrm{S}^{-}}$ bands. Far infrared photometry was obtained from the AllWISE data release (Cutri et al. 2014) in the four WISE-bands.

The Galactic reddening $E(B-V)$ and the Galactic dust extinction $A_{V}$ from the maps of Schlafly \& Finkbeiner (2011) are also provided. However, correcting for reddening and extinction only works properly for stars situated significantly above the Galactic plane. Bright hot subdwarfs or stars at low Galactic latitudes are usually foreground objects.

The extended wavelength coverage of the multi-band photometry allows us to construct full spectral energy distributions of both single $\mathrm{sdO} / \mathrm{Bs}$ and $\mathrm{sdO} / \mathrm{Bs}$ in binaries with cool companions. SEDs are a powerful tool to determine their parameters (e.g., Girven et al. 2012). The results of the SED fitting of all the stars in the catalog will be published in another paper of this series (see Fig. 2 for some examples).

\subsection{Ground-based proper motions}

Ground-based proper motions were obtained from SDSS (DR9, Ahn et al. 2012), the Fourth US Naval Observatory CCD Astrograph Catalog (UCAC4, Zacharias et al. 2013), the 

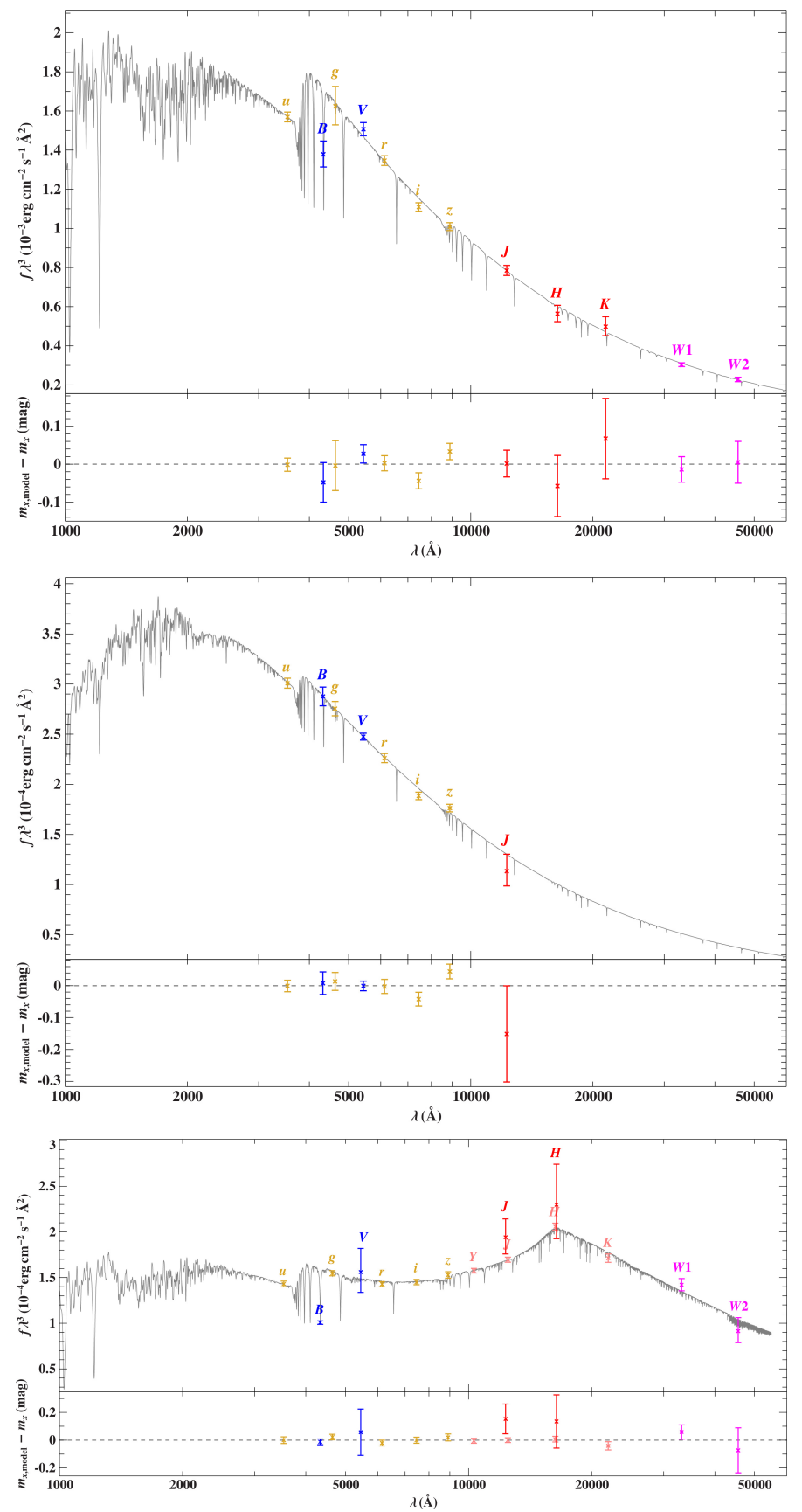

Fig. 2. Preliminary spectral energy distributions (SEDs) and residuals for an sdB star with $T_{\text {eff }} \simeq 29500 \mathrm{~K}$ (upper panel), an sdO star with $T_{\text {eff }} \simeq 39700 \mathrm{~K}$ (middle panel) and a composite binary system consisting of an sdB star with $T_{\text {eff }} \simeq 29100 \mathrm{~K}$ and a K-type main sequence star with $T_{\text {eff }} \simeq 4500 \mathrm{~K}$ (lower panel). Photometry is taken from the catalog. The $u$-band is especially important for determining reliable effective temperatures for $\mathrm{sdO} / \mathrm{Bs}$. The fitting procedure with synthetic models is described in Irrgang (2014).

PPMXL catalog of positions and proper motions on the ICRS (Roeser et al. 2010), the Whole-Sky USNO-B1.0 Catalog (Monet et al. 2003), the Absolute Proper motions Outside the Plane catalog (APOP, Qi et al. 2015), and the Yale/San Juan Southern Proper Motion Catalog 4 (SPM4, Girard et al. 2011).

\subsection{Light-curve data}

To search for variability likely caused by close binarity (e.g. eclipses, reflection effects, ellipsoidal modulations) or pulsations, available light curves of all the stars in the SuperWASP archive (DR1, Butters et al. 2010) and the Catalina Real-Time Transient Survey (CRTS DR2, Drake et al. 2012) were visually inspected. The SuperWASP DR1 only covers a small fraction of the objects in the catalog and is restricted to bright stars ( $<15 \mathrm{mag})$. The light curves usually have several thousand single epochs. The CRTS DR2 has a significant overlap with our catalog and covers the magnitude range $\sim 13-20 \mathrm{mag}$. The number of epochs varies from a few tens to a few hundred.

Due to the limited quality of the light curves and strong aliasing effects, we refrained from performing a rigorous statistical analysis of the light curves. Instead the implemented periodogram functions of the Catalina and the NASA Exoplanet Archive web pages were used to search for significant periodicities (keyword var). In addition, all light curves were visually checked for irregular variations (var irregular). It has to be pointed out that only relatively strong variations can be seen in these data and that objects marked as constant in the catalog can still show lower amplitude variations.

The catalog contains comments about the availability and the properties of the light curves for all stars. Candidates for periodic variabilities come with the tentative period in days (e.g., $\operatorname{var}$ 0.123). However, only variabilities that are marked with the keyword strong should be treated as reliable. The other candidates might well be marginal and require a more sophisticated analysis. We found a variety of new close binary and pulsator candidates, which will be published by Kupfer et al. (in prep.).

\subsection{Cleaning the catalog}

The data collected were used to identify and remove objects misclassified as hot subdwarf stars. Likely candidates are hot white dwarfs of DA, DB and DO type, peculiar objects such as PG 1159 stars and central stars of planetary nebula, but also cooler DAs, which show weaker hydrogen lines and can resemble helium-poor sdBs (especially if spectra are normalized to a flat continuum). Main sequence stars of $\mathrm{O}$ and $\mathrm{B}$ type as well as blue $\mathrm{HB}$ (BHB) stars are possible bright contaminants. Cool subdwarfs of $A$ and $F$ type as well as cataclysmic variables can mimic $\mathrm{sdO} / \mathrm{B}+\mathrm{MS}$ binaries. Extragalactic objects such as blue galaxies and QSOs also appear in color-selected samples. To separate all kinds of cool objects, color indices were used. Approximately 300 objects with SDSS colors $u-g>0.6$ and $g-r>0.1$, as well as $N U V_{\mathrm{GALEX}}-g_{\mathrm{APASS}}>2.0$, have been excluded.

A powerful tool to separate nearby white dwarfs from the more luminous and distant $\mathrm{sdO} / \mathrm{Bs}$ is the reduced proper motion. We followed the approach outlined in Gentile Fusillo et al. (2015) and calculated the reduced proper motion $H=x+$ $5 \log \mu+5$. The full proper motion $\mu$ was averaged from all available proper motions of each star. Since not all stars have photometry in all bands, we defined the magnitude $x$ as the average of all the blue and visual bands in the catalog $\left(B j_{\mathrm{GSC}}, V_{\mathrm{GSC}}, B_{\mathrm{GSC}}\right.$, $\left.V_{\mathrm{APASS}}, B_{\mathrm{APASS}}, g_{\mathrm{APASS}}, u_{\mathrm{SDSS}}, g_{\mathrm{SDSS}}, u_{\mathrm{VST}}, g_{\mathrm{VST}}\right)$. It has to be pointed out that this is not a physically meaningful quantity, but an empirically determined filter parameter only. For the subset of stars with SDSS photometry, we compared $H$ with the reduced proper motion in the $g$-band $H_{\mathrm{g}}=g+5 \log \mu+5$. The mean deviation is \pm 0.12 , which can become much higher $(>0.5)$ for stars with incomplete photometry. 


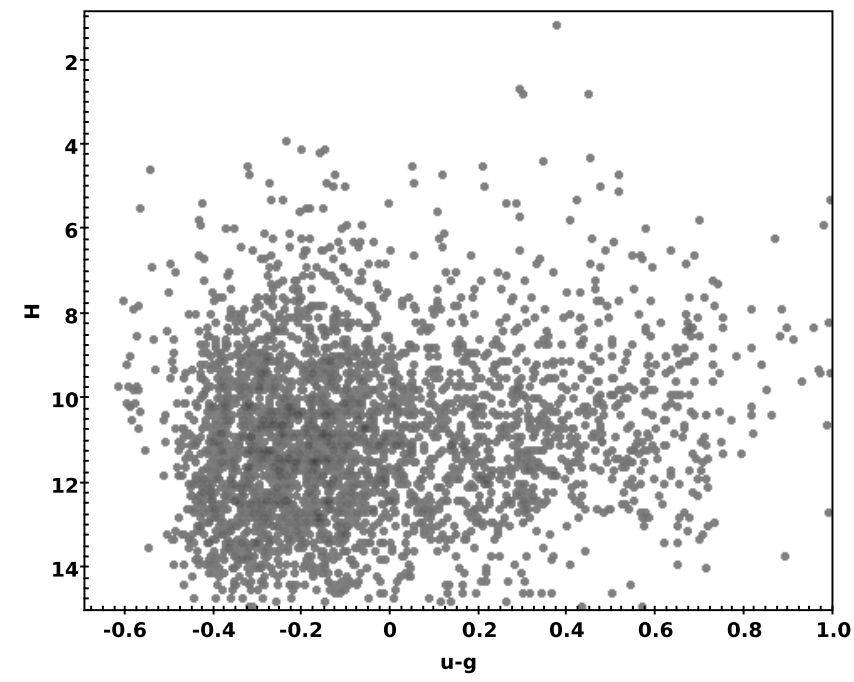

Fig. 3. Reduced proper motion $H$ as defined in Sect. 2.5 for a subset of stars with $u-g$ colors. Outliers with inconsistent proper motions between the different proper motion catalogs have been included in our catalog, but are not shown here.

We constructed the reduced proper motion diagram and also included previously misclassified white dwarfs (WDs) to find the most reasonable exclusion criterion (see Fig. 3). We found that stars with $H>15$ are very likely to be WDs (see also Gentile Fusillo et al. 2015) and excluded them. Exceptions have been made for stars with highly inconsistent proper motions.

The light curves also turned out to be useful to identify misclassifications. Strong irregular variations led to the exclusion of some cataclysmic variables (CVs) and the characteristic pulsations of RR Lyr variables are easy to recognize and allowed us to exclude a few tens of those stars.

The catalog was also cross-matched with SIMBAD and misclassified objects known from the literature (mostly B-stars, WDs, CVs, and central stars of planetary nebula) were also excluded. Another benefit of the various cross-matching exercises was the identification of a few tens of stars (some of them very bright) with incorrect coordinates in SIMBAD. We corrected those coordinates in the catalog by adopting either SDSS or 2MASS positions.

Finally, we inspected the distribution of values in the single catalog columns as well as the two color diagrams with TOPCAT to find and eliminate obvious outliers. However, the large amount of heterogeneous archive data has not been systematically checked for quality (e.g. by inspecting quality flags). The final catalog contains 5613 unique objects, most of which are located in the northern hemisphere and at relatively high Galactic latitudes, because most surveys cover those regions (see Fig. 4). Besides some WDs and MS-B stars we expect BHB stars to be the most important class of contaminant objects remaining in the catalog, because they are very hard to separate from sdO/Bs with the methods used here.

The first preliminary data from the Gaia mission has been published recently (Gaia collaboration 2016). However, due to yet uncorrected chromatic effects, the bluest objects have been excluded from Gaia Data Release 1. Only approximately 60 stars of our catalog are part of the bright TGAS sample (Tycho-Gaia astrometric solution, Michalik et al. 2015) with preliminary parallaxes and proper motions, almost all of them composite $\mathrm{sdB}$ binaries. The much larger Gaia DR1 catalog of positions and Gaia magnitudes only covers approximately half of our sample.
Due to this severe selection bias we decided not to include Gaia DR1 data in this version of our catalog.

\subsection{Classification of hot subdwarfs}

Most of the stars in the catalog have been visually classified by looking at some kind of optical spectrum (objective prism, longslit, echelle). It is almost impossible to homogenize the classifications attributed by different people at different times based on very different types of data. Furthermore, quite diverse classification schemes have emerged in the last few decades, from the quite detailed classifications in the PG catalog (Green et al. 1986) and the more general classes introduced (and widely used today) by Moehler et al. (1990) to the sophisticated MK-like classification system developed by Drilling et al. (2013).

The spectroscopic classifications provided for 5055 stars in the catalog (see Table 1) follow the scheme outlined in Moehler et al. (1990). Classifications from the literature have been adapted to this scheme. The reason is that statistical analyses of population properties require a meaningful definition of the sub-populations rather than overly detailed classifications. The exact spectroscopic class might still be interesting to study single objects. In this case, the catalog links to the literature can be used to find out more.

It has to be pointed out that some subclasses are relatively difficult to distinguish spectroscopically. The only difference between the sdBs and sdOBs is often the presence or absence of only one He II line at $4686 \AA$, which can be easily overlooked in low-quality spectra. The helium-rich subclasses (He-sdB, especially $\mathrm{He}$-sdOB and $\mathrm{He}$-sdO) can often only be distinguished with a proper quantitative spectral analysis. Stars classified as $\mathrm{sdO}$ in the catalog can be both hydrogen- or helium-rich, because they are often not clearly separated in the literature.

Assuming that the spectroscopic classifications are, on average, correct, we used them to define a purely empirical scheme for the photometric classifications by inspecting the locations of the subclasses in two-color diagrams (see Table 2). Using the SDSS $u-g$ versus $g-r$ diagram (Fig. 5, see also Geier et al. 2011), sdOs of all types are well separated from the sdBs. sd+MS stars, on the other hand, form a distinct sequence and are clearly separated from the cooler stars of A and F-type, which have been excluded. The sd+MS sequence is also well defined in the $N U V_{\mathrm{GALEX}}-g_{\mathrm{APASS}}$ versus $g_{\mathrm{APASS}}-r_{\mathrm{APASS}}$ diagram (Fig. 6 , upper panel), whereas the hotter sdOs cannot be separated. To provide at least an approximate photometric class of the remaining objects, we also used the less well defined color criterium $V_{\mathrm{GSC}}-J_{2 \mathrm{MASS}}$ (Fig. 6, lower panel) to distinguish between single $\mathrm{sdO} / \mathrm{Bs}$ and composite sd+MS systems. The SDSS-based color class should be regarded the most trustworthy, followed by the GALEX/APASS class and eventually the GSC/2MASS class. Following this ranking, we defined a comprehensive color class and classified 4888 stars in the catalog in this way.

The color classes can also be used as a sanity check for the spectroscopic classifications, e.g. to find yet undetected main sequence companions, and of course are very useful for a tentative classification of stars without spectroscopy.

\subsection{Spectroscopic parameters and radial velocities}

The catalog contains spectroscopic parameters such as effective temperatures, surface gravities, and helium abundances for 924 stars from the literature. This fraction is not complete, because only papers containing larger samples of sdO/B stars 
S. Geier et al.: The population of hot subdwarf stars studied with Gaia. I.

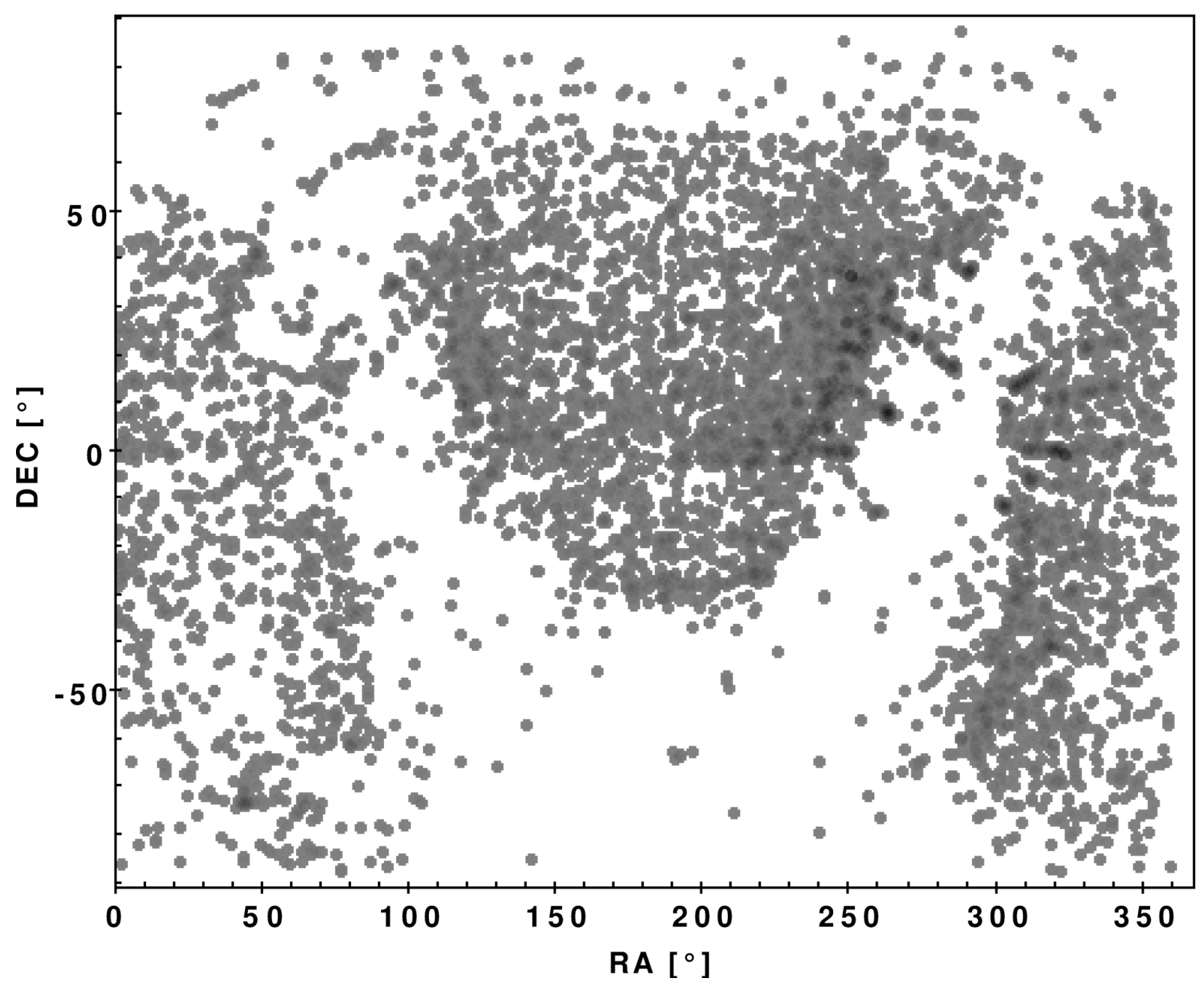

Fig. 4. Coordinates of the objects in the hot subdwarf catalog.

Table 1. Spectral classification scheme.

\begin{tabular}{ll}
\hline \hline sdB & H, (He I) \\
sdOB & H, He I, He II \\
sdO & H, He II (H-rich) or He II, (H) (He-rich) \\
$\mathrm{He}-$ sdB & He I, (H) \\
$\mathrm{He}-$-sdOB & He I, He II, (H) \\
$\mathrm{He}-\mathrm{sdO}$ & $\mathrm{He}$ II, (He I, H) \\
sdO/B+K/G/F/A & sdO/B with visible MS companion \\
\hline sdO/BV & Pulsator \\
sdO/B+WD/dM/BD & sdO/B with close invisible companion \\
\hline
\end{tabular}

Notes. For each class the species with observed lines in the spectrum are given in a sequence of relative strength. The strongest lines are always coming first. Species in parentheses can be present, but do not have to be.

have been taken into account (Heber et al. 1984; Bixler et al. 1991; Saffer et al. 1994, 1997; Maxted et al. 2001; Edelmann et al. 2003; Lisker et al. 2005; Ströer et al. 2007; Hirsch 2009; Østensen et al. 2010a; Nemeth et al. 2012; Geier et al. 2013, 2015b; Kupfer et al. 2015; Luo et al. 2016; Kepler et al. 2016).

Radial velocities (RVs) are provided for the 2122 stars with spectra in the SDSS data archive. Those RVs have been measured automatically by cross-correlation with template spectra
Table 2. Color-classification schemes.

\begin{tabular}{ll}
\hline \hline $\begin{array}{l}\text { Color class } 1 \\
\text { SDSS }\end{array}$ & \\
\hline sdO & $-0.55<u_{\mathrm{SDSS}}-g_{\mathrm{SDSS}}<-0.35$ \\
& $-0.65<g_{\mathrm{SDSS}}-r_{\mathrm{SDSS}}<-0.45$ \\
sdB & $-0.5<u_{\mathrm{SDSS}}-g_{\mathrm{SDSS}}<0.7$ \\
& $g_{\mathrm{SDSS}}-r_{\mathrm{SDSS}}>0.208\left(u_{\mathrm{SDSS}}-g_{\mathrm{SDSS}}\right)-0.516$ \\
& $g_{\mathrm{SDSS}}-r_{\mathrm{SDSS}}<0.208\left(u_{\mathrm{SDSS}}-g_{\mathrm{SDSS}}\right)-0.376$ \\
sd+MS & $-0.5<u_{\mathrm{SDSS}}-g_{\mathrm{SDSS}}<0.7$ \\
& $g_{\mathrm{SDSS}}-r_{\mathrm{SDSS}}>0.208\left(u_{\mathrm{SDSS}}-g_{\mathrm{SDSS}}\right)-0.376$ \\
\hline Color class 2 & \\
GALEX/APASS & \\
\hline sdO/B & $N U V_{\mathrm{GALEX}}-g_{\mathrm{APASS}}<2.0$ \\
sd+MS & $g_{\mathrm{APASS}}-r_{\mathrm{APASS}}<-0.15$ \\
& $N U V_{\mathrm{GALEX}}-g_{\mathrm{APASS}}<2.0$ \\
\hline Color class 3 & $g_{\mathrm{APASS}}-r_{\mathrm{APASS}} \geq-0.15$ \\
GSC/2MASS & \\
\hline sdO/B & \\
sd+MS & $V_{\mathrm{GSC}}-J_{2 \mathrm{MASS}}<0.1$ \\
\hline
\end{tabular}

in the course of the SDSS. Since the template library does not include very helium-rich stars such as He-sdOs, the RVs of such 

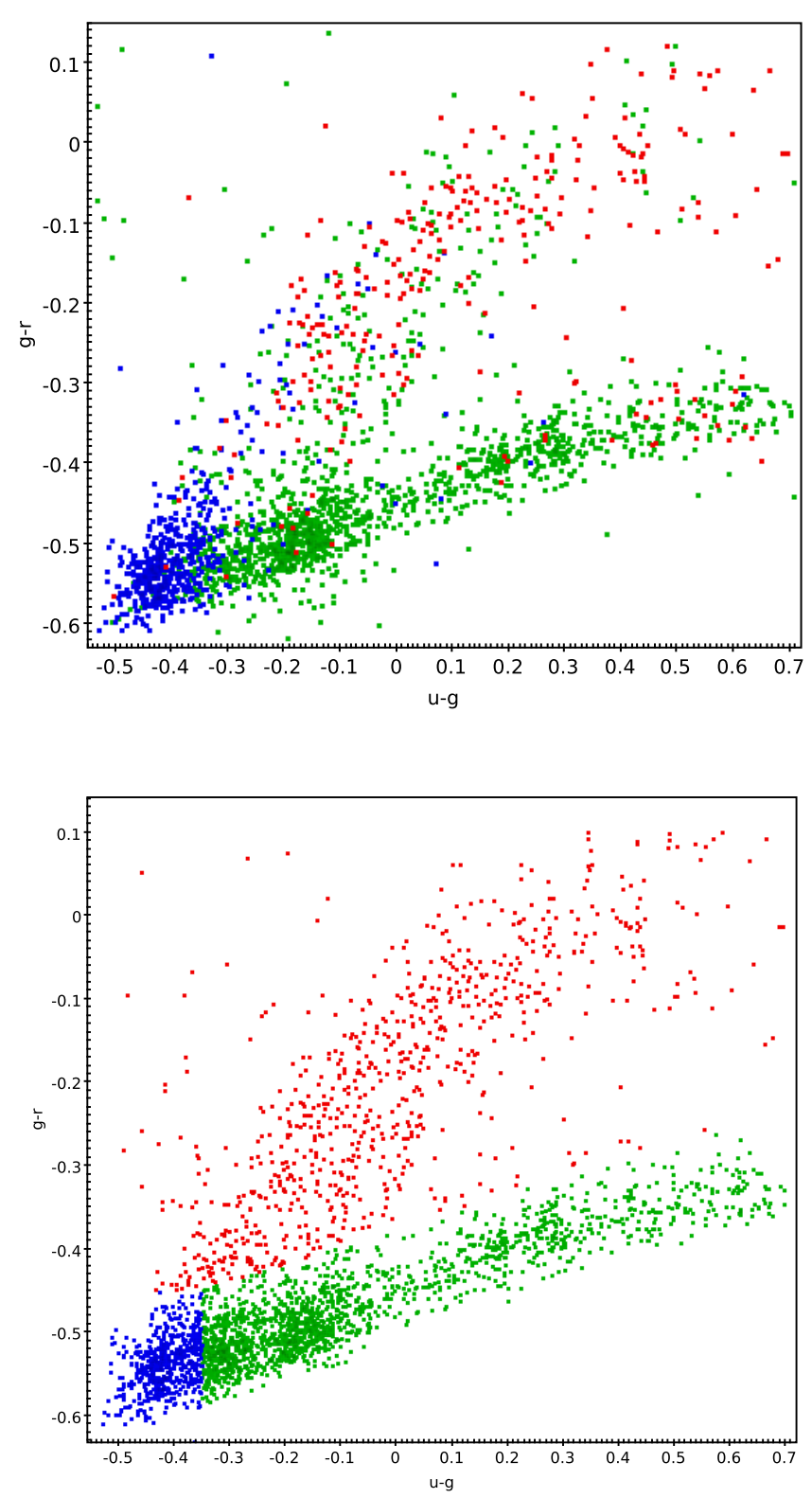

Fig. 5. Two-color diagram for the stars with SDSS color classifications. The color indices have been corrected for reddening as described in Schlafly \& Finkbeiner (2011). Stars classified in this way as sdOs are marked in blue, sdBs in green and composite sd+MS systems in red. Upper panel: stars with spectroscopic classifications. The color cuts in the lower panel are determined from this plot. It can be clearly seen that composite systems are easier to identify from their colors than from their spectra.

stars can be incorrect by up to $\sim 100 \mathrm{~km} \mathrm{~s}^{-1}$. The reason for this is that the Pickering series of single ionized helium can easily be confused with the hydrogen Balmer series. However, the rest wavelengths of the Pickering lines are blueshifted with respect to the Balmer lines. Fitting a He-sdO with a normal O star template can therefore lead to an apparently good match, but result in the wrong RV. For He-sdOs with pure helium atmospheres this effect is strongest and must be taken into account (see Geier et al. 2015a).

The RVs are obtained from SDSS spectra, which are usually co-added from three consecutive individual exposures of $15 \mathrm{~min}$ duration. Since especially sdB stars are often found in close binaries with sometimes very short orbital periods, orbital smearing
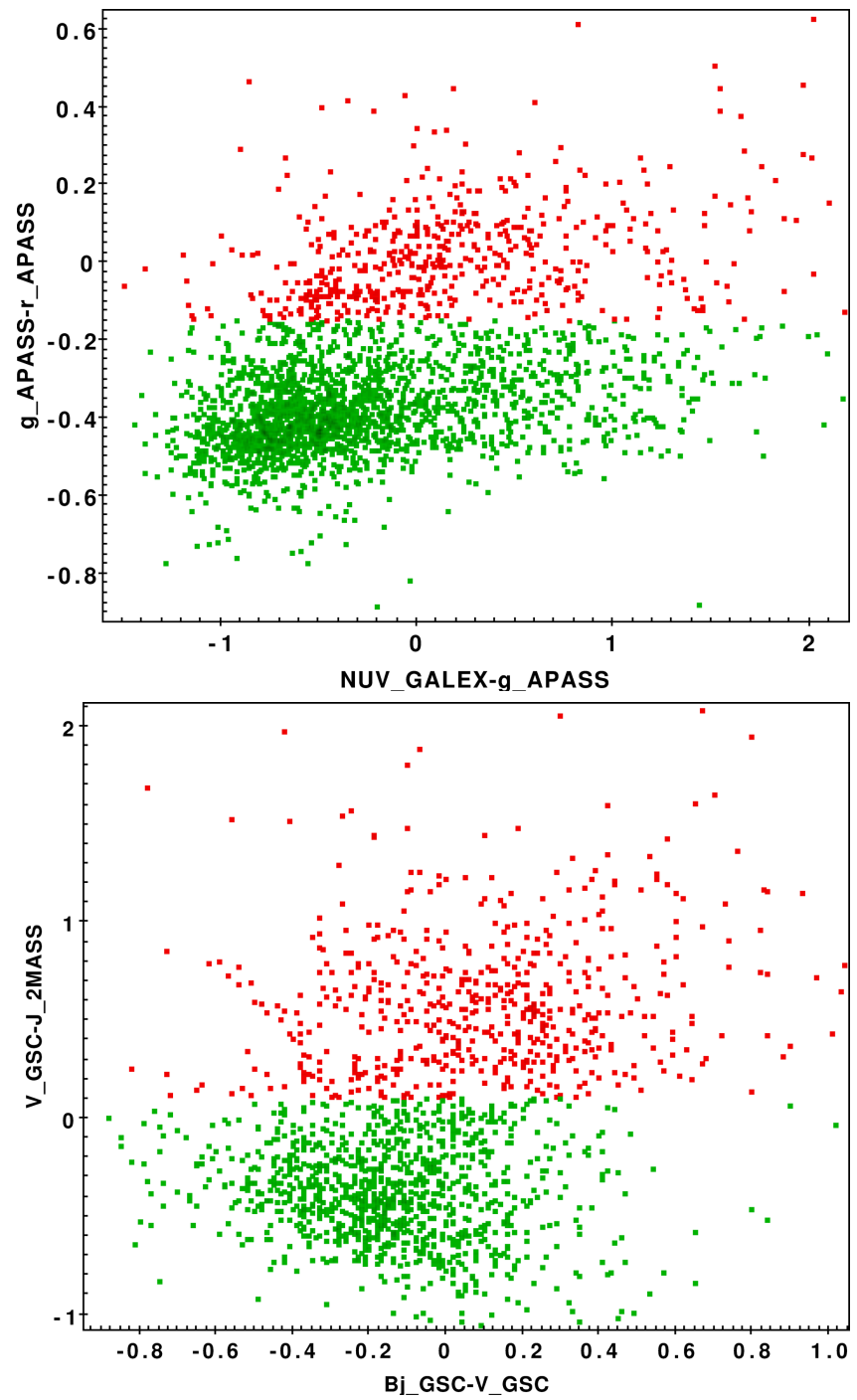

Fig. 6. Two-color diagrams for the stars with GALEX/APASS (upper panel) and GSC/2MASS (lower panel) color classifications. Because those stars are on average brighter and closer, no reddening correction has been applied. Stars classified in this way as sdO/Bs are marked in green and composite sd+MS systems in red.

can lead to systematic shifts (e.g. Geier et al. 2011). More importantly, the SDSS RVs in this catalog are only obtained from one co-added spectrum and contain no information about RV variability (as provided for sdO/Bs up to SDSS DR7 in Geier et al. 2015b).

\subsection{Catalog statistics and data access}

Table A.1 summarizes the content of the catalog. Since the sample is heterogeneously selected, those numbers only contain very limited information about the quantitative properties of the underlying Galactic hot subdwarf population. The most striking difference between the spectroscopic and the color selection is the much higher number of composite sd+MS systems found by colors. Part of this mismatch can very likely be attributed to the quality of the photometry and the selection of the color cuts.

However, considering that most spectral classifications are based on blue and visual spectra, where the cool companion is quite often outshone by the $\mathrm{sdO} / \mathrm{B}$ component, infrared excesses 
Table 3. Catalog statistics.

\begin{tabular}{ll}
\hline \hline Total & 5613 \\
\hline Spectroscopic class & \\
\hline sdB & 2866 \\
sdOB & 530 \\
sdO & 477 \\
He-sdB/OB/O & 540 \\
sd+MS & 642 \\
\hline Unclassified sd & 558 \\
\hline Color class & \\
\hline sdB & 1666 \\
sdO & 548 \\
sdO/B & 1448 \\
sd+MS & 1226 \\
\hline Unclassified sd & 725 \\
\hline
\end{tabular}

are a much better indication for a cool companion than the sometimes quite shallow spectral lines (e.g. Nemeth et al. 2016), we conclude that the color selection should be better suited to find such binaries, especially if the companions are of late type, and that the fraction of those systems might have been underestimated so far.

The catalog will be available via the VizieR service as well as the German Virtual Observatory (GAVO). A detailed description of the catalog columns is provided in Table A.1. It will be linked to all relevant databases to allow easy access to data for individual objects. The catalog is by no means complete and heterogeneously selected, which has to be taken into account when using it for statistical analyses. People who are interested in individual objects should carefully check the relevant quality flags in the specific catalogs.

If an object is in the catalog, there is a high chance that it will indeed be a hot subdwarf star. However, some misclassified objects are certainly still there. If a hot subdwarf candidate from one of the input samples is not in the catalog, it is extremely likely, that it is not a hot subdwarf star, because it did not pass our selection criteria. Combining the color classes with the spectroscopic classification it is possible to select purer subsamples.

\section{Conclusions}

The new catalog of hot subdwarfs presented here contains a significant fraction of the total $\mathrm{sdO} / \mathrm{B}$ population with apparent visual magnitudes between $\sim 9$ mag and $\sim 20 \mathrm{mag}$, which translates into distances from a few tens pc to more than $20 \mathrm{kpc}$ and therefore includes stars from the thin disk, thick disk, and halo population. This reasonably well characterized sample will be cross-matched with the Gaia catalog and used to define the criteria (reduced proper motions, distances, color cuts in the Gaia bands, etc.) for the selection of a homogeneous all-sky catalog of $\mathrm{sdO} / \mathrm{B}$ stars.

It will become an important input catalog for ground-based light curve transient and transit surveys such as the Palomar Transient Factory (PTF), the BlackGEM and Gravitational-wave Optical Transient Observer (GoTo) surveys for optical counterparts of gravitational wave transients, the Next Generation Transit Survey for exoplanets (NGTS), or the Large Synoptic Survey Telescope (LSST), but also space missions such as the ongoing K2 mission, the PLAnetary Transits and Oscillations of stars (PLATO) mission, the Transiting Exoplanet Survey Satellite (TESS), the Wide Field Infrared Survey Telescope (WFIRST), and the Euclid mission. Due to the high fraction of close binaries in the hot subdwarf population, we expect to find many of those based on their characteristic light curve variations.

We are also planning to use the catalog as input for widearea spectroscopic surveys such as LAMOST, the WEAVE survey at La Palma, the 4-m Multi-Object Spectroscopic Telescope (4MOST) survey in Chile, and the Dark Energy Spectroscopic Instrument (DESI) survey and obtain spectroscopy for a large fraction of the stars. With a density of less than one object per square degree on average, only very few fibres would be necessary to carry out a fairly complete survey as a side project to the main surveys.

Besides the extension of the catalog and the inclusion of the Gaia data, other important datasets such as the multi-band, widearea surveys PanSTARRS and SkyMapper will be included in future releases.

Acknowledgements. We want to thank the referee Dave Kilkenny for his constructive report and for reminding us not to neglect the southern hemisphere. The research leading to these results has received funding from the European Research Council under the European Union's Seventh Framework Programme (FP/2007-2013)/ERC Grant Agreement No. 320964 (WDTracer). This research made use of TOPCAT, an interactive graphical viewer and editor for tabular data Taylor (2005). This research made use of the SIMBAD database, operated at CDS, Strasbourg, France; the VizieR catalog access tool, CDS, Strasbourg, France. Some of the data presented in this paper were obtained from the Mikulski Archive for Space Telescopes (MAST). STScI is operated by the Association of Universities for Research in Astronomy, Inc., under NASA contract NAS526555. Support for MAST for non-HST data is provided by the NASA Office of Space Science via grant NNX13AC07G and by other grants and contracts. This research has made use of the services of the ESO Science Archive Facility. This publication makes use of data products from the Two Micron All Sky Survey, which is a joint project of the University of Massachusetts and the Infrared Processing and Analysis Center/California Institute of Technology, funded by the National Aeronautics and Space Administration and the National Science Foundation. Based on observations made with the NASA Galaxy Evolution Explorer. GALEX is operated for NASA by the California Institute of Technology under NASA contract NAS5-98034. This research has made use of the APASS database, located at the AAVSO web site. Funding for APASS has been provided by the Robert Martin Ayers Sciences Fund. The Guide Star Catalog-II is a joint project of the Space Telescope Science Institute and the Osservatorio Astronomico di Torino. Space Telescope Science Institute is operated by the Association of Universities for Research in Astronomy, for the National Aeronautics and Space Administration under contract NAS5-26555. The participation of the Osservatorio Astronomico di Torino is supported by the Italian Council for Research in Astronomy. Additional support is provided by European Southern Observatory, Space Telescope European Coordinating Facility, the International GEMINI project and the European Space Agency Astrophysics Division. Based on observations obtained as part of the VISTA Hemisphere Survey, ESO Program, 179.A-2010 (PI: McMahon). This publication has made use of data from the VIKING survey from VISTA at the ESO Paranal Observatory, programme ID 179.A-2004. Data processing has been contributed by the VISTA Data Flow System at CASU, Cambridge and WFAU, Edinburgh. Based on data products from observations made with ESO Telescopes at the La Silla Paranal Observatory under program ID 177.A 3011(A,B,C,D,E.F). Based on data products from observations made with ESO Telescopes at the La Silla Paranal Observatory under programme IDs 177.A-3016, 177.A-3017 and 177.A-3018, and on data products produced by Target/OmegaCEN, INAF-OACN, INAF-OAPD and the KiDS production team, on behalf of the KiDS consortium. OmegaCEN and the KiDS production team acknowledge support by NOVA and NWO-M grants. Members of INAF-OAPD and INAF-OACN also acknowledge the support from the Department of Physics \& Astronomy of the University of Padova, and of the Department of Physics of Univ. Federico II (Naples). This publication makes use of data products from the Wide-field Infrared Survey Explorer, which is a joint project of the University of California, Los Angeles, and the Jet Propulsion Laboratory/California Institute of Technology, and NEOWISE, which is a project of the Jet Propulsion Laboratory/California Institute of Technology. WISE and NEOWISE are funded by the National Aeronautics and Space Administration. Based on observations made with the Nordic Optical Telescope, operated by the Nordic Optical Telescope Scientific Association at the Observatorio del Roque de los Muchachos, La Palma, Spain, of the Instituto de Astrofisica de Canarias. Based 
on observations at the La Silla-Paranal Observatory of the European Southern Observatory. Based on observations collected at the Centro Astronómico Hispano Alemán (CAHA) at Calar Alto, operated jointly by the Max-Planck Institut für Astronomie and the Instituto de Astrofísica de Andalucía (CSIC). Based on observations with the William Herschel and Isaac Newton Telescopes operated by the Isaac Newton Group at the Observatorio del Roque de los Muchachos of the Instituto de Astrofisica de Canarias on the island of La Palma, Spain. Based on observations at Kitt Peak National Observatory, National Optical Astronomy Observatory, which is operated by the Association of Universities for Research in Astronomy (AURA) under cooperative agreement with the National Science Foundation. The authors are honored to be permitted to conduct astronomical research on Iolkam Du'ag (Kitt Peak), a mountain with particular significance to the Tohono O'odham. Funding for the SDSS and SDSS-II has been provided by the Alfred P. Sloan Foundation, the Participating Institutions, the National Science Foundation, the US Department of Energy, the National Aeronautics and Space Administration, the Japanese Monbukagakusho, the Max Planck Society, and the Higher Education Funding Council for England. The SDSS Web Site is http: //www. sdss.org/. The SDSS is managed by the Astrophysical Research Consortium for the Participating Institutions. The Participating Institutions are the American Museum of Natural History, Astrophysical Institute Potsdam, University of Basel, University of Cambridge, Case Western Reserve University, University of Chicago, Drexel University, Fermilab, the Institute for Advanced Study, the Japan Participation Group, Johns Hopkins University, the Joint Institute for Nuclear Astrophysics, the Kavli Institute for Particle Astrophysics and Cosmology, the Korean Scientist Group, the Chinese Academy of Sciences (LAMOST), Los Alamos National Laboratory, the Max-Planck-Institute for Astronomy (MPIA), the Max-Planck-Institute for Astrophysics (MPA), New Mexico State University, Ohio State University, University of Pittsburgh, University of Portsmouth, Princeton University, the United States Naval Observatory, and the University of Washington. Funding for SDSS-III has been provided by the Alfred P. Sloan Foundation, the Participating Institutions, the National Science Foundation, and the US Department of Energy Office of Science. The SDSSIII web site is http://www.sdss3.org/. SDSS-III is managed by the Astrophysical Research Consortium for the Participating Institutions of the SDSS-III Collaboration including the University of Arizona, the Brazilian Participation Group, Brookhaven National Laboratory, University of Cambridge, Carnegie Mellon University, University of Florida, the French Participation Group, the German Participation Group, Harvard University, the Instituto de Astrofisica de Canarias, the Michigan State/Notre Dame/JINA Participation Group, Johns Hopkins University, Lawrence Berkeley National Laboratory, Max Planck Institute for Astrophysics, Max Planck Institute for Extraterrestrial Physics, New Mexico State University, New York University, Ohio State University, Pennsylvania State University, University of Portsmouth, Princeton University, the Spanish Participation Group, University of Tokyo, University of Utah, Vanderbilt University, University of Virginia, University of Washington, and Yale University.

\section{References}

Ahn, C. P., Alexandroff, R., Allende Prieto, C., et al. 2012, ApJS, 203, 21 Alam, S., Albareti, F. D., Allende Prieto, C., et al. 2015, ApJS, 219, 12 Bianchi, L., Herald, J., Efremova, B., et al. 2011, Ap\&SS, 335, 161 Bixler, J. V., Bowyer, S., \& Laget, M. 1991, A\&A, 250, 370

Butters, O. W., West, R. G., Anderson, D. R., et al. 2010, A\&A, 520, A10

Camarota, L., \& Holberg, J. B. 2014, MNRAS, 438, 3111

Chavira, E. 1958, Bol. Obs. Tonantz. Tacub., 2, 15

Chavira, E. 1959, Bol. Obs. Tonantz. Tacub., 2, 3

Cutri, R. M., et al. 2014, VizieR Online Catalog: II/328

de Jong, J. T. A., Verdoes Klejin, G. A., Boxhoorn, D., et al. 2015, A\&A, 582, 62

Downes, R. A. 1986, ApJS, 61, 569

Drake, A. J., Djorgovski, S. G., Mahabal, A., et al. 2012, IAU Symp., 285, 306

Drilling, J. S., Jeffery, C. S., Heber, U., Moehler, S., \& Napiwotzki, R. 2013, A\&A, 551, A31

Edge, A., Sutherland, W., Kuijken, K., et al. 2013, The Messenger, 154, 32
Edelmann, H., Heber, U., Hagen, H.-J., et al. 2003, A\&A, 400, 939 Gaia Collaboration (Brown, A.G.A, et al.) 2016, A\&A, 595, A2 Geier, S. 2015, Astron. Narch., 336, 437

Geier, S., Hirsch, H., Tillich, A., et al. 2011, A\&A, 530, 28

Geier, S., Heber, U., Edelmann, H., et al. 2013, A\&A, 557, 122

Geier, S., Fürst, F., Ziegerer, E., et al. 2015a, Science, 347, 1126

Geier, S., Kupfer, T., Heber, U., et al. 2015b, A\&A, 577, A26

Gentile Fusillo, N. P., Gänsicke, B. T., \& Greiss, S. 2015, MNRAS, 448, 2260

Girard, T. M., van Altena, W. F., Zacharias, N., et al. 2011, AJ, 142, 15

Girven, J., Steeghs, D., Heber, U., et al. 2012, MNRAS, 425, 1013

Green, R. F., Schmidt, M., \& Liebert, J. 1986, ApJS, 61, 305

Hagen, H.-J., Groote, D., Engels, D., \& Reimers, D. 1995, A\&AS, 111, 195

Haro, G., \& Luyten, W. J. 1962, Bol. Obs. Tonantz. Tacub., 3, 37

Heber, U. 1986, A\&A, 155, 33

Heber, U. 2016, PASP, 128, 082001

Heber, U., Hunger, K., Jonas, G., \& Kudritzki, R. P. 1984, A\&A, 130, 119

Henden, A. A., Templeton, M., Terrell, D., et al. 2016, VizieR Online Data Catalog: II/336

Hirsch, H. 2009, Ph.D. Thesis, Friedrich-Alexander University ErlangenNürnberg

Humason, M. L., \& Zwicky, F. 1947, ApJ, 105, 85

Iriarte, B., \& Chavira, E. 1957, Bol. Obs. Tonantz. Tacub., 2, 3

Irrgang, A. 2014, Ph.D. Thesis, Friedrich-Alexander University ErlangenNürnberg

Kepler, S. O., Pelisoli, I., Koester, D., et al. 2015, MNRAS, 446, 4078

Kepler, S. O., Pelisoli, I., Koester, D., et al. 2016, MNRAS, 455, 3413

Kilkenny, D., Heber, U., \& Drilling, J. S. 1988, South African Astron. Obs. Circ, 12,1

Kilkenny, D., O’Donoghue, D., Worters, H. L., et al. 2015, MNRAS, 453, 1879

Kilkenny, D., Worters, H. L., O'Donoghue, D., et al. 2016, MNRAS, 459, 4343

Kupfer, T., Geier, S., Schaffenroth, V., et al. 2015, A\&A, 576, 44

Lasker, B. M., Lattanzi, M. G., McLean, B. J., et al. 2008, AJ, 136, 735

Lawrence, A., Warren, S. J., Almaini, O., et al. 2007, MNRAS, 379, 1599

Lisker, T., Heber, U., Napiwotzki, R., et al. 2005, A\&A, 430, 223

Luo, Y.-P., Nemeth, P., Liu, C., Deng, L.-C., \& Han, Z. 2016, ApJ, 818, 202

Maxted, P. F. L., Heber, U., Marsh, T. R., \& North, R. C. 2001, MNRAS, 326, 139

Michalik, D., Lindegren, L., \& Hobbs, D. 2015, A\&A, 574, A115

Mickaelian, A. M. 2008, AJ, 136, 946

Mickaelian, A. M., Nesci, R., Rossi, C., et al. 2007, A\&A, 464, 1177

Moehler, S., Richtler, T., de Boer, K. S., Dettmar, R. J., \& Heber, U. 1990, A\&AS, 86, 53

Monet, D. G., Levine, S. E., Canzian, B., et al. 2003, AJ, 125, 984

Nemeth, P., Kawka, A., \& Vennes, S. 2012, MNRAS, 427, 2180

Nemeth, P., Ziegerer, E., Irrgang, A., et al. 2016, ApJ, 821, L13

O'Donoghue, D., Kilkenny, D., Koen, C., et al. 2013, MNRAS, 431, 240

Oreiro, R., Rodriguez-Lopez, C., Solano, E., et al. 2011, A\&A, 530, A2

Østensen, R. H. 2006, Baltic Astron., 15, 85

Østensen, R. H., Oreiro, R., Solheim, J.-E., et al. 2010a, A\&A, 513, A6

Østensen, R. H., Silvotti, R., Charpinet, S., et al. 2010b, MNRAS, 409, 1470

Perez-Fernandez, E., Ulla, A., Solano, E., Oreiro, R., \& Rodrigo, C. 2016, MNRAS, 457, 3396

Qi, Z., Yu, L., Bucciarelli, B., et al. 2015, AJ, 150, 137

Roeser, S., Demleitner, M., \& Schilbach, E. 2010, AJ, 139, 2440

Saffer, R. A., Bergeron, P., Koester, D., \& Liebert, J. 1994, ApJ, 432, 351

Saffer, R. A., Keenan, F. P., Hambly, N. C., Dufton, P. L., \& Liebert, J. 1997, ApJ, 491, 172

Schlafly, E. F., \& Finkbeiner, D. P. 2011, ApJ, 737, 103

Shanks, T., Metcalfe, N., Chehade, B., et al. 2015, MNRAS, 451, 4238

Skrutskie, M. F., Cutri, R. M., Stiening, R., et al. 2006, AJ, 131, 1163

Stobie, R. S., Kilkenny, D., O’Donoghue, D., et al. 1997, MNRAS, 287, 848

Ströer, A., Heber, U., Lisker, T., et al. 2007, A\&A, 462, 269

Taylor, M. B. 2005, ASP Conf. Ser., 347, 29

Vennes, S., Kawka, A., \& Németh, P. 2011, MNRAS, 410, 2095

Wisotzki, L., Koehler, T., Groote, D., \& Reimers, D. 1996, A\&AS, 115, 227

Zacharias, N., Finch, C. T., Girard, T. M., et al. 2013, AJ, 145, 44 


\section{Appendix A: Additional table}

Table A.1. Catalog columns.

\begin{tabular}{|c|c|c|c|}
\hline Column & Format & Description & Unit \\
\hline NAME & A30 & Target name & \\
\hline RA & F10.6 & Right ascension (J2000) & $\operatorname{deg}$ \\
\hline DEC & F10.6 & Declination (J2000) & $\operatorname{deg}$ \\
\hline SPEC_CLASS & A15 & Spectroscopic classification & \\
\hline COLOR_CLASS1 & A 10 & Color classification SDSS & \\
\hline COLOR_CLASS2 & A10 & Color classification GALEX/APASS & \\
\hline COLOR_CLASS3 & A10 & Color classification GSC/2MASS & \\
\hline COLOR_CLASS & A 10 & Color classification & \\
\hline RV_SDSS & F5.1 & Radial velocity SDSS & $\mathrm{km} \mathrm{s}^{-1}$ \\
\hline e_RV_SDSS & F5.1 & Error on RV_SDSS & $\mathrm{km} \mathrm{s}^{-1}$ \\
\hline T_EFF & F8.1 & Effective temperature & $\mathrm{K}$ \\
\hline e_T_EFF & F8.1 & Error on T_EFF & $\mathrm{K}$ \\
\hline LOG_G & $\mathrm{F} 4.2$ & Log surface gravity (gravity in $\mathrm{cm} \mathrm{s}^{-2}$ ) & $\operatorname{dex}$ \\
\hline e_LOG_G & F.4.2 & Error on LOG_G & $\operatorname{dex}$ \\
\hline LOG_Y & F5.2 & Log helium abundance $n(\mathrm{He}) / n(\mathrm{H})$ & $\operatorname{dex}$ \\
\hline e_LOG_Y & F5.2 & Error on LOG_Y & $\operatorname{dex}$ \\
\hline PARAMS_REF & A20 & Reference for atmospheric parameters (Bibcode) & \\
\hline EB-V & F6.4 & Instellar reddening $E(B-V)$ & mag \\
\hline e_EB-V & F6.4 & Error on $E B-V$ & mag \\
\hline AV & F6.4 & Instellar extinction $A_{V}$ & mag \\
\hline FUV_GALEX & F6.3 & GALEX $F U V$-band magnitude & mag \\
\hline e_FUV_GALEX & F6.3 & Error on FUV_GALEX & mag \\
\hline NUV_GALEX & F6.3 & GALEX $N U V$-band magnitude & mag \\
\hline e_NUV_GALEX & F6.3 & Error on NUV_GALEX & mag \\
\hline FUV_GALEX_CORR & F6.3 & GALEX FUV-band magnitude corrected (Camarota \& Holberg 2014) & mag \\
\hline NUV_GALEX_CORR & F6.3 & GALEX $N U V$-band magnitude corrected (Camarota \& Holberg 2014) & mag \\
\hline F_GSC & F6.3 & Guide Star Catalog $R_{F}$-band magnitude & mag \\
\hline e_F_GSC & F6.3 & Error on F_GSC & mag \\
\hline Bj_GSC & F6.3 & Guide Star Catalog $B_{j}$-band magnitude & mag \\
\hline e_Bj_GSC & F6.3 & Error on $\mathrm{Bj} \_\mathrm{GSC}$ & mag \\
\hline V_GSC & F6.3 & Guide Star Catalog $V$-band magnitude & mag \\
\hline e_V_GSC & F6.3 & Error on V_GSC & mag \\
\hline N_GSC & F6.3 & Guide Star Catalog $I_{N}$-band magnitude & mag \\
\hline e_N_GSC & F6.3 & Error on N_GSC & mag \\
\hline B_GSC & F6.3 & Guide Star Catalog $B$-band magnitude & mag \\
\hline e_B_GSC & F6.3 & Error on B_GSC & mag \\
\hline V_APASS & F6.3 & APASS $V$-band magnitude & mag \\
\hline e_V_APASS & F6.3 & Error on V_APASS & mag \\
\hline B_APASS & F6.3 & APASS $B$-band magnitude & mag \\
\hline e_B_APASS & F6.3 & Error on V_APASS & mag \\
\hline g_APASS & F6.3 & APASS $g$-band magnitude & mag \\
\hline e_g_APASS & F6.3 & Error on g_APASS & mag \\
\hline r_APASS & F6.3 & APASS $r$-band magnitude & mag \\
\hline e_r_APASS & F6.3 & Error on r_APASS & mag \\
\hline i_APASS & F6.3 & APASS $i$-band magnitude & mag \\
\hline e_i_APASS & F6.3 & Error on i_APASS & mag \\
\hline u_SDSS & F6.3 & SDSS $u$-band magnitude & mag \\
\hline e_u_SDSS & F6.3 & Error on u_SDSS & mag \\
\hline g_SDSS & F6.3 & SDSS $g$-band magnitude & mag \\
\hline e_g_SDSS & F6.3 & Error on g_SDSS & mag \\
\hline
\end{tabular}


Table A.1. continued.

\begin{tabular}{|c|c|c|c|}
\hline Column & Format & Description & Unit \\
\hline r_SDSS & F6.3 & SDSS $r$-band magnitude & mag \\
\hline e_r_SDSS & F6.3 & Error on r_SDSS & mag \\
\hline i_SDSS & F6.3 & SDSS $i$-band magnitude & mag \\
\hline e_i_SDSS & F6.3 & Error on i_SDSS & mag \\
\hline z_SDSS & F6.3 & SDSS $z$-band magnitude & mag \\
\hline e_z_SDSS & F6.3 & Error on z_SDSS & mag \\
\hline u_VST & F6.3 & VST surveys (ATLAS, KiDS) $u$-band magnitude & mag \\
\hline e_u_VST & F6.3 & Error on u_VST & mag \\
\hline g_VST & F6.3 & VST surveys (ATLAS, KiDS) $g$-band magnitude & mag \\
\hline e_g_VST & F6.3 & Error on g_VST & mag \\
\hline r_VST & F6.3 & VST surveys (ATLAS, KiDS) $r$-band magnitude & mag \\
\hline e_r_VST & F6.3 & Error on r_VST & mag \\
\hline i_VST & F6.3 & VST surveys (ATLAS, KiDS) $i$-band magnitude & mag \\
\hline e_i_VST & F6.3 & Error on i_VST & mag \\
\hline z_VST & F6.3 & VST surveys (ATLAS, KiDS) $z$-band magnitude & mag \\
\hline e_z_VST & F6.3 & Error on $z_{-}$VST & mag \\
\hline J_2MASS & F6.3 & 2MASS $J$-band magnitude & mag \\
\hline e_J_2MASS & F6.3 & Error on J_2MASS & mag \\
\hline H_2MASS & F6.3 & 2MASS $H$-band magnitude & mag \\
\hline e_H_2MASS & F6.3 & Error on H_2MASS & mag \\
\hline K_2MASS & F6.3 & 2MASS $K$-band magnitude & mag \\
\hline e_K_2MASS & F6.3 & Error on K_2MASS & mag \\
\hline Y_UKIDSS & F6.3 & UKIDSS $Y$-band magnitude & mag \\
\hline e_Y_UKIDSS & F6.3 & Error on Y_UKIDSS & mag \\
\hline J_UKIDSS & F6.3 & UKIDSS $J$-band magnitude & mag \\
\hline e_J_UKIDSS & F6.3 & Error on J_UKIDSS & mag \\
\hline H_UKIDSS & F6.3 & UKIDSS $H$-band magnitude & mag \\
\hline e_H_UKIDSS & F6.3 & Error on H_UKIDSS & mag \\
\hline K_UKIDSS & F6.3 & UKIDSS $K$-band magnitude & mag \\
\hline e_K_UKIDSS & F6.3 & Error on K_UKIDSS & mag \\
\hline Z_VISTA & F6.3 & VISTA surveys (VHS, VIKING) Z-band magnitude & mag \\
\hline e_Z_VISTA & F6.3 & Error on Z_VISTA & mag \\
\hline Y_VISTA & F6.3 & VISTA surveys (VHS, VIKING) $Y$-band magnitude & mag \\
\hline e_Y_VISTA & F6.3 & Error on Y_VISTA & mag \\
\hline J_VISTA & F6.3 & VISTA surveys (VHS, VIKING) $J$-band magnitude & mag \\
\hline e_J_VISTA & F6.3 & Error on J_VISTA & mag \\
\hline H_VISTA & F6.3 & VISTA surveys (VHS, VIKING) $H$-band magnitude & mag \\
\hline e_H_VISTA & F6.3 & Error on H_VISTA & mag \\
\hline Ks_VISTA & F6.3 & VISTA surveys (VHS, VIKING) $K s$-band magnitude & mag \\
\hline e_Ks_VISTA & F6.3 & Error on Ks_VISTA & mag \\
\hline W1 & F6.3 & WISE $W 1$-band magnitude & mag \\
\hline e_W1 & F6.3 & Error on W1 & mag \\
\hline W2 & F6.3 & WISE W2-band magnitude & mag \\
\hline e_W2 & F6.3 & Error on W2 & mag \\
\hline W3 & F6.3 & WISE W3-band magnitude & mag \\
\hline e_W3 & F6.3 & Error on W3 & mag \\
\hline W4 & F6.3 & WISE W4-band magnitude & mag \\
\hline e_W4 & F6.3 & Error on W4 & mag \\
\hline PM_RA_SDSS & F6.1 & SDSS proper motion $\mu_{\alpha} \cos \delta$ & mas $\mathrm{yr}^{-1}$ \\
\hline e_PM_RA_SDSS & F6.1 & Error on PM_RA_SDSS & $\operatorname{mas} \mathrm{yr}^{-1}$ \\
\hline PM_DEC_SDSS & F6.1 & SDSS proper motion $\mu_{\delta}$ & mas $\mathrm{yr}^{-1}$ \\
\hline e_PM_DEC_SDSS & F6.1 & Error on PM_DEC_SDSS & mas $\mathrm{yr}^{-1}$ \\
\hline
\end{tabular}


S. Geier et al.: The population of hot subdwarf stars studied with Gaia. I.

Table A.1. continued.

\begin{tabular}{|c|c|c|c|}
\hline Column & Format & Description & Unit \\
\hline PM_RA_UCAC4 & F6.1 & UCAC4 proper motion $\mu_{\alpha} \cos \delta$ & $\operatorname{mas} \mathrm{yr}^{-1}$ \\
\hline e_PM_RA_UCAC4 & F6.1 & Error on PM_RA_UCAC4 & mas $\mathrm{yr}^{-1}$ \\
\hline PM_DEC_UCAC4 & F6.1 & UCAC4 proper motion $\mu_{\delta}$ & mas $\mathrm{yr}^{-1}$ \\
\hline e_PM_DEC_UCAC4 & F6.1 & Error on PM_DEC_UCAC4 & mas $\mathrm{yr}^{-1}$ \\
\hline PM_RA_PPMXL & F6.1 & PPMXL proper motion $\mu_{\alpha} \cos \delta$ & $\operatorname{mas} \mathrm{yr}^{-1}$ \\
\hline e_PM_RA_PPMXL & F6.1 & Error on PM_RA_PPMXL & mas $\mathrm{yr}^{-1}$ \\
\hline PM_DEC_PPMXL & F6.1 & PPMXL proper motion $\mu_{\delta}$ & mas $\mathrm{yr}^{-1}$ \\
\hline e_PM_DEC_PPMXL & F6.1 & Error on PM_DEC_PPMXL & mas $\mathrm{yr}^{-1}$ \\
\hline PM_RA_USNO & F6.1 & USNO B1.0 proper motion $\mu_{\alpha} \cos \delta$ & mas $\mathrm{yr}^{-1}$ \\
\hline e_PM_RA_USNO & F6.1 & Error on PM_RA_USNO & mas $\mathrm{yr}^{-1}$ \\
\hline PM_DEC_USNO & F6.1 & USNO B1.0 proper motion $\mu_{\delta}$ & mas $\mathrm{yr}^{-1}$ \\
\hline e_PM_DEC_USNO & F6.1 & Error on PM_DEC_USNO & $\operatorname{mas} \mathrm{yr}^{-1}$ \\
\hline PM_RA_APOP & F6.1 & APOP proper motion $\mu_{\alpha} \cos \delta$ & mas $\mathrm{yr}^{-1}$ \\
\hline e_PM_RA_APOP & F6.1 & Error on PM_RA_APOP & mas $\mathrm{yr}^{-1}$ \\
\hline PM_DEC_APOP & F6.1 & APOP proper motion $\mu_{\delta}$ & mas $\mathrm{yr}^{-1}$ \\
\hline e_PM_DEC_APOP & F6.1 & Error on PM_DEC_APOP & mas $\mathrm{yr}^{-1}$ \\
\hline PM_RA_SPM4 & F6.1 & SPM4 proper motion $\mu_{\alpha} \cos \delta$ & mas $\mathrm{yr}^{-1}$ \\
\hline e_PM_RA_SPM4 & F6.1 & Error on PM_RA_SPM4 & $\operatorname{mas} \mathrm{yr}^{-1}$ \\
\hline PM_DEC_SPM4 & F6.1 & SPM4 proper motion $\mu_{\delta}$ & mas $\mathrm{yr}^{-1}$ \\
\hline e_PM_DEC_SPM4 & F6.1 & Error on PM_DEC_SPM4 & mas $\mathrm{yr}^{-1}$ \\
\hline LC_CRTS & A30 & CRTS light curve properties & \\
\hline LC_SWASP & A30 & SWASP light curve properties & \\
\hline
\end{tabular}

